\title{
Caso Clínico: Cáncer de Tiroides de Etiología Sarcoma en Pediatría
}

\section{Clinical Case: Thyroid Cancer of Etiology Sarcoma in Pediatrics}

*Correspondencia: drzambrano2013@yahoo.com Teléfono [593] 2281744

Conflicto de intereses: Los autores declaran no tener conflictos de intereses.

Fondos: Ver la página 81

Recibido: 1 Febrero 2018 Aceptado: 11 Diciembre 2018 Publicado: 30 Abril 2019

\section{Membrete bibliográfico:} Leone $\mathrm{M}$, Zambrano C. Caso clínico: Cáncer de Tiroides de Etiología Sarcoma en Pediatría. Rev. Oncol. Ecu 2019;29(1):74-82.

ISSN: 2661-6653

DOI: https://doi.org/10.33821/258

Copyright Leone, et al. Este artículo es distribuido bajo los términos de Creative Commons Attribution License, el cual permite el uso y redistribución citando la fuente y al autor original.

\section{Mario Leone Pignataro1(D), Cecilio Zambrano García1*(iD)}

1. Servicio de Partes Blandas, SOLCA - Guayaquil.

\section{Resumen}

Introducción: El cáncer de tiroides es de presentación poco frecuente en pacientes pediátricos, que en lo general dependiendo del tipo celular tiene una evolución favorable posterior a cirugía e iodoradioablacion. En el Instituto Oncológico Nacional se registró una incidencia de 11 casos en menores de 15 años entre el 2010 y 2016.

Caso Clínico: se presenta el caso de un hombre de 13 años con diagnóstico de sarcoma de tiroides de tipo cartilaginoso, con marcador tumoral KI67 positivo, de presentación agresiva e invasión ganglionar regional y metástasis, con presentación inicial de lesión tumoral de gran volumen en región tiroidea, acompañada de disnea y disfagia.

Evolución: El plan quirúrgico oncológico fue citorreducción, seguido de un esquema de quimioterapia y radioterapia con el objetivo de aumentar su expectativa de vida.

Conclusión: Los sarcomas son tumores poco frecuentes, representando menos del $1 \%$ de todas las neoplasias. Se presenta este caso, porque existen pocos reportes a nivel mundial.

Palabras Claves: NEOPLASIAS DE LA TIROIDES, NIÑO, SARCOMA

DOI: $10.33821 / 258$

\section{Abstract}

Introduction: Thyroid cancer is a rare presentation in pediatric patients, which in general depending on the cell type has a favorable evolution after surgery and iodoradioablacion. In the National Oncology Institute there was an incidence of 11 cases in children under 15 years between 2010 and 2016.

Clinical case: the case of a 13-year-old man diagnosed with cartilage-type thyroid sarcoma, with positive KI67 tumor marker, aggressive presentation and regional lymph node invasion and metastasis, with initial presentation of large volume tumor lesion in the region thyroid, accompanied by dyspnea and dysphagia.

Evolution: The oncological surgical plan was cytoreduction, followed by a chemotherapy and radiotherapy scheme with the aim of increasing their life expectancy. 
Conclusion: Sarcomas are rare tumors, representing less than $1 \%$ of all neoplasms. This case is presented, because there are few reports worldwide.

Keywords: THYROID NEOPLASMS, CHILD, SARCOMA

DOI: $10.33821 / 258$

\section{Introducción}

El cáncer de tiroides (CT) es de presentación poco frecuente en Pacientes Pediátricos (PP). En Estados Unidos según el programa Surveillance, Epidemiology and End Results (SEER), los nuevos casos en personas menores de 20 años representan el $1.8 \%$ de todas las neoplasias malignas diagnosticadas de tiroides [1]. En el Instituto Oncológico Nacional (ION), se registra una incidencia de 11 casos en menores de 15 años durante el periodo 2010 al 2016 [2]. En este reporte se describirá un caso de sarcoma debido a su baja incidencia en el ION.

La frecuencia de esta patología está aumentando entre los adolescentes de 15 a 19 años, el CT es el octavo cáncer diagnosticado con mayor frecuencia y el segundo cáncer más común entre las niñas. Los adolescentes tienen una incidencia 10 veces mayor que los niños más pequeños y durante la adolescencia hay una relación de mujer a hombre (5:1) que no se observa en niños pequeños. La presentación más común es la de un nódulo tiroideo [3, 4].

La clasificación patológica de los CT en los PP se basa en las definiciones establecidas por la Organización Mundial de la Salud (OMS), con los criterios histológicos iguales para niños y adultos. El cáncer papilar de tiroides (CPT) representa el $90 \%$ o más de todos los casos infantiles. El cáncer folicular de tiroides (CFT) es poco frecuente, mientras que el cáncer medular de tiroides (CMT), los tumores poco diferenciados y los carcinomas de tiroides francamente indiferenciados (anaplásicos) son raros [5, 6]. El tratamiento de esta enfermedad se establece inicialmente con una tiroidectomía radical (TR) seguida de radioiodoablación [7, 8] (RIA).

El objetivo del presente reporte es describir este interesante caso y hacer una revisión de las principales características, técnica quirúrgica, y esquema de tratamiento. El mismo que es multidisciplinario generando controversia por su efectividad y seguridad.

\section{Caso clínico}

Se trata de un hombre de 13 años de edad atendido en el ION por presentar masa en región de glándula tiroides (GT) de 5 meses de evolución, con biopsia de nódulo en lóbulo derecho de GT reportando sarcoma fusocelular de bajo grado con diferenciación cartilaginosa. 
Al examen físico se apreció una cicatriz en el triángulo anterior del cuello, producto de una intervención previa. A la palpación de detalló una masa sólida, fija no móvil. El paciente presentó disnea de moderados esfuerzos (Figura 1).

La valoración con una Tomografía Contrastada de cuello reportó una masa heterogénea, la cual involucraba ambos lóbulos de la GT y el istmo, con áreas de baja densidad y calcificaciones, con crecimiento hacia el mediastino superior, y endoluminal a la vía aérea superior, con oclusión de la misma en aproximadamente el $80 \%$, con infiltración de cartílagos tiroideos, con una medición de $100 \mathrm{~mm}$ de longitud y $80 \mathrm{~mm}$ en su eje transverso (Figura 2, 3).

Figura 1. Examen físico del cuello.

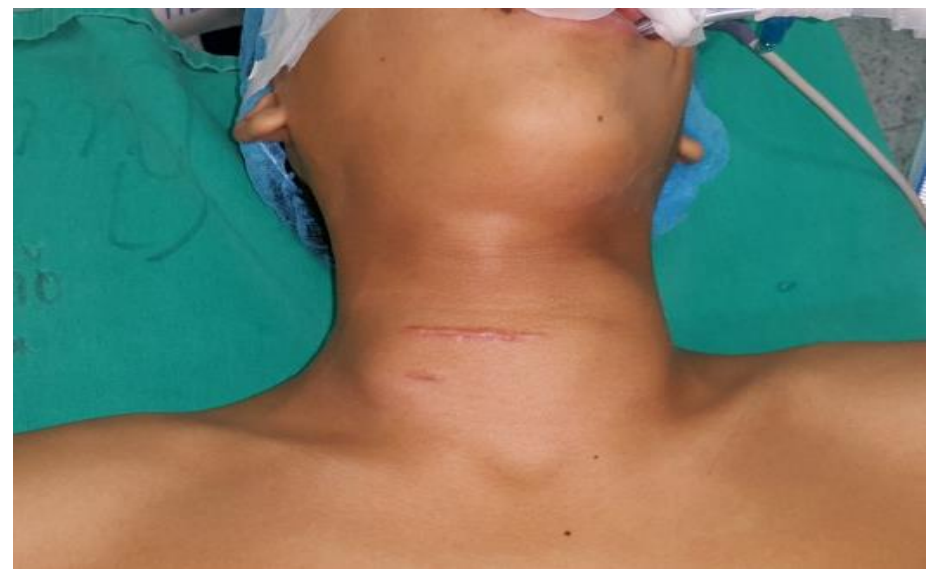

Imagen de la masa tumoral como distorsiona y desplaza las estructuras del cuello.

Figura 2. Resonancia Magnética de cuello, corte sagital

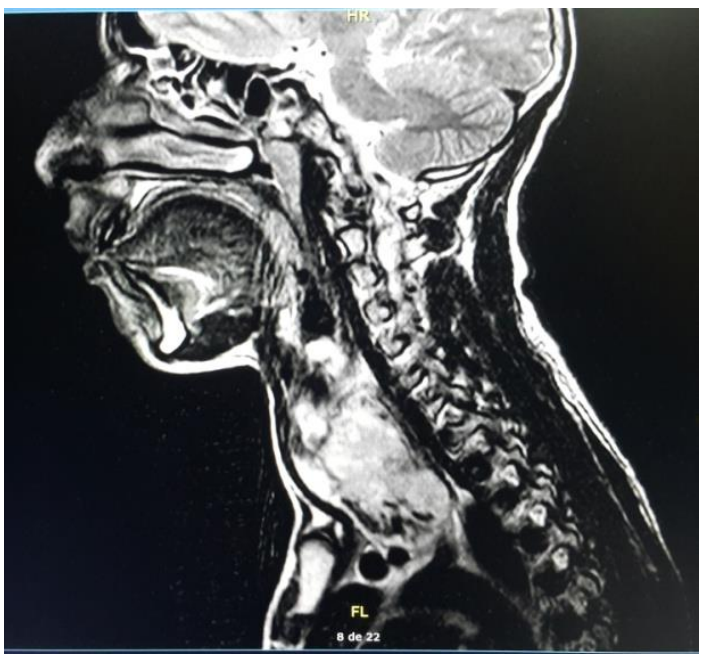

RM de cabeza y cuello corte sagital y coronal de un paciente de 13 años con diagnóstico de Sarcoma de Tiroides, en el que se evidencia masa heterogénea, que involucra ambos lóbulos de la glándula tiroides y el istmo. 
Figura 3. Resonancia Magnética de cuello, corte coronal

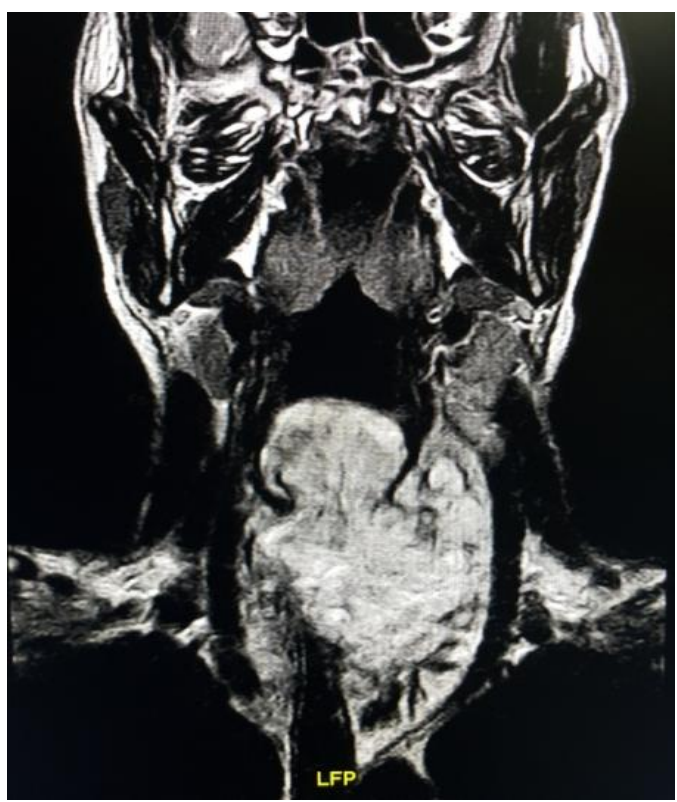

El estudio demuestra áreas de baja densidad y calcificaciones, con crecimiento hacia el mediastino superior, y endoluminal a la vía aérea superior, con oclusión de la misma en aproximadamente $70-80 \%$, con infiltración de los cartílagos tiroideos, aritenoideos, cricoideo, espacio retrofaringeo, y topografía de cuerdas vocales, mide aprox. $100 \mathrm{~mm}$ de longitud y $80 \mathrm{~mm}$ en su eje transverso

\section{Figura 4. Exploración del cuello}

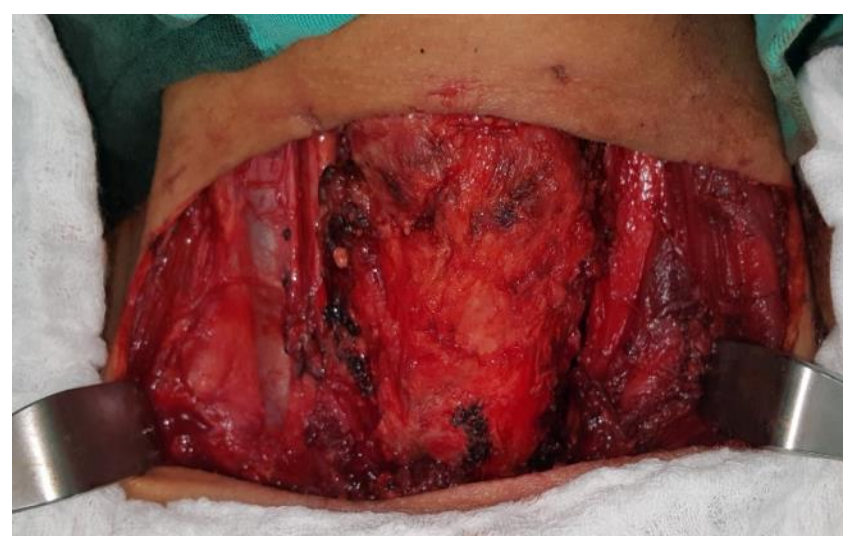

Previa diéresis de cervicotomía anterior extendida se identifica la lesión tumoral, la cual estaba adherida a la tráquea y esófago, realizándose traqueotomía y gastrostomía. 
Figura 5. Estudio Histológico

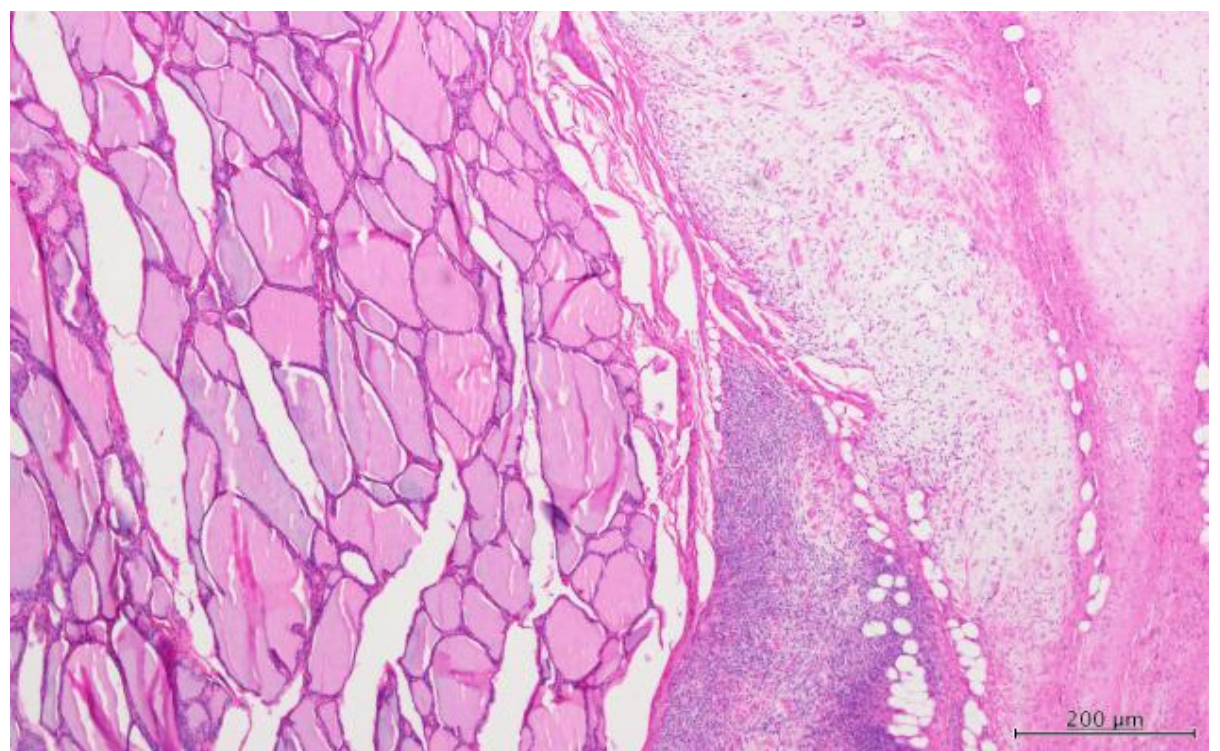

Microscopía (Tinción con hematoxilina-eoxina 10x) en la cual se aprecia el folículo tiroideo con el cambio a tejido cartilaginoso.

\section{Figura 6. Estudio Histológico}

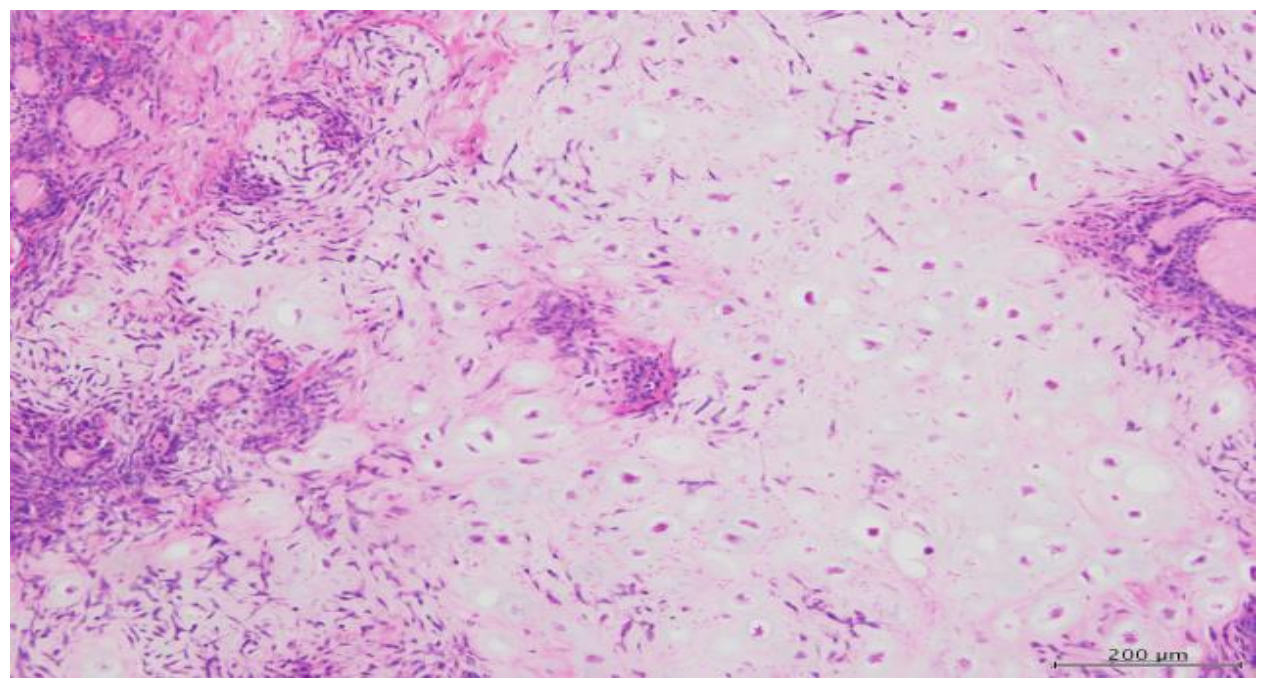

Microscopía (Tinción con hematoxilina-eoxina 40x) que muestra sarcoma fusocelular de bajo grado con diferenciación cartilaginosa.

Por la complejidad del caso el comité de tumores de tiroides sugirió la realización de una cervicotomia exploratoria con intención de citoreducción paliativa y la realización de una traqueotomía y gastrostomía (Figura 4). 
El estudio anatomopatológico post-quirúrgico reportó un Sarcoma Fusocelular de bajo grado con diferenciación cartilaginosa (Figura 5, 6). La muestra se estudió en el Departament of Pathology, Medical College Of Wisconsin.

Se realizaron inmunotinciones de: citoqueratina AE1/AE3: negativo, P63: negativo, SMA: negativo, DESMINA: negativo, CD-10: negativo, TTF1: negativo, S-100: positivo, BCL2: positivo, CD-99: positivo, SOX 10: negativo. HMB45: negativo, CD117: negativo, CD-31: negativo, $\mathrm{KI}-67$ : positivo $10-15 \%$.

\section{Evolución}

El paciente inició el tratamiento con ciclos de quimioterapia (QTP): Ifosfamida $1950 \mathrm{mg}+$ Mesna $390 \mathrm{mg}$ + Ondasetron $6.5 \mathrm{mg}$ + Cardioxane $210 \mathrm{mg}$ + Doxorrubicina $21 \mathrm{mg}$ y radioterapia (RTP) 6 ciclos. El último control del paciente fue en junio del 2017 encontrándose en condiciones regulares, y con dificultad para caminar por la presencia de metástasis óseas en la extremidad inferior izquierda.

\section{Discusión}

Los datos epidemiológicos de CT en pediátricos, predomina el sexo femenino, con un pico en adolescentes, asociado a síndromes endócrinos, tumoración cervical, ganglios cervicales o metástasis pulmonares [9-11]. Los sarcomas de partes blandas son tumores poco frecuentes, que representan menos del $1 \%$ de todas las neoplasias. En el caso se destaca en la valoración inicial el crecimiento de la región tiroidea con afectación de ganglios regionales.

Los métodos diagnósticos de evaluación inicial incluyen la tiroglobulina y calcitonina que aportan información sugestiva de lesión tiroidea. La Asociación Americana Clínica de Endocrinología [12] reporta para la PAAF una sensibilidad del $83 \%$, especificidad del $92 \%$ y valor predictivo positivo del $75 \%$. La ecografía se considera la primera prueba de cribado en el estudio del nódulo tiroideo, seguido de la TC y resonancia magnética, los cuales contribuyeron a definir la infiltración del CT con sus metástasis en la progresión de la enfermedad. El marcador tumoral Ki-67 es un marcador del nivel de replicación celular, y se expresa como índice de proliferación, se apreció en un 10 a $15 \%$ en la histopatología del caso lo que guió al diagnóstico de sarcoma.

El tratamiento inicial es quirúrgico, la experiencia reportada de otros estudios refieren que la TR con disección selectiva de los ganglios linfáticos es el tratamiento a seguir. La meta es lograr una remisión completa $[9,11,13]$, siempre que proporcionen unos márgenes, tanto laterales como profundos, libres de enfermedad microscópica. Se deben recalcar las complicaciones posoperatorias como: hipoparatiroidismo $(4.5-17 \%)$, parálisis del recurrente laríngeo (1.3-6 \%), Síndrome de Horner (0.4\%) o infección de la herida quirúrgica $(0.1 \%)[14]$, y considerar que al ser pediátricos el impacto que influirá en su calidad de vida. 
La indicación de RIA es destruir focos malignos de enfermedad microscópica y residual, por lo que se recomienda su uso después de TR [15]. El tratamiento estándar de primera línea para sarcomas en los pacientes con buen estado general (de 0 a 2 en la escala de la OMS) es la doxorrubicina [9].

El pronóstico del CT es bueno con una tasa de sobrevida mayor del $90 \%$. Las revisiones retrospectivas $[9,11,15]$ recalcan como factores riesgo: presentación en edad temprana, tamaño del tumor primario con extensión extratiroidea, ganglios linfáticos palpables, presencia de metástasis distantes, cirugía incompleta, supresión insuficiente de hormona tiroidea, histología poco diferenciada con células atípicas, invasión folicular y necrosis. Condiciones importantes de recalcar en el caso del sarcoma de tipo cartilaginoso con un pronóstico malo por la presentación avanzada del mismo.

\section{Conclusiones}

Los sarcomas son tumores de gran agresividad y de sintomatología muy variada. En este reporte se caracterizó por una invasión extensa del tumor al momento del diagnóstico, con pronósticos reservados. Es importante insistir en valoraciones precoces ante la presencia de nódulos tiroideos o aumento de la GT en la etapa pediátrica como parte de la valoración de rutina del niño sano.

\section{Agradecimientos}

Agradecemos a los padres del paciente que permitieron la publicación del presente caso.

Nota del Editor

La Revista Oncología Ecu

permanece neutral con respecto a los reclamos jurisdiccionales en mapas publicados y afiliaciones institucionales.

\section{Información adicional}

\section{Abreviaturas}

CFT: cáncer folicular de tiroides

CMT: cáncer medular de tiroides

CPT: cáncer papilar de tiroides

CT: Cáncer de Tiroides.

GT: Glándula Tiroides.

OMS: Organización Mundial de la Salud

PAAF: Punción Aspirativa de Aguja Fina.

PP: Pacientes Pediátricos.

QTP: Quimiterapia.

RIA: radioiodoablación

TR: tiroidectomía radical 
Archivos Adicionales

Ninguno declarado por los autores.

Fondos

Los fondos de la investigación fueron propios de los autores del presente artículo.

Disponibilidad de datos y materiales

Existe la disponibilidad de datos bajo solicitud al autor de correspondencia. No se reportan otros materiales.

\section{Contribuciones de los autores}

$M L P, C Z G$, participaron igual en idea de investigación, revisión bibliográfica, recolección de datos, escritura del artículo. MLP, realizó las correcciones editoriales. Todos los autores leyeron y aprobaron la versión final del artículo.

Aprobación de ética y consentimiento para participar

No aplica a este estudio.

\section{Consentimiento para publicación}

Se cuenta con la autorización escrita por parte los padres del paciente para la publicación del caso con fines académicos.

\section{Información de los autores}

Mario Leone Pignataro, Jefe del Servicio de Partes Blandas y Sarcomas- Cirujano Oncólogo

- Docente de Post grado Universidad Católica de Guayaquil, Universidad Guayaquil, Universidad de Especialidades “Espíritu Santo" (UEES).

Cecilio Zambrano García, Médico Especialista en Cirugía General - Magíster en Gerencia y Administración en Salud. 


\section{Referencias}

Abreviaturas en la referencias

DOI: Digital Object Identifier

PMID: PubMed Identifier

SU: Short URL
1. Noone AM, Howlader N, Krapcho M, Miller D, Brest A, Yu M, et al. SEER Cancer Statistics Review, 19752015, National Cancer Institute. Bethesda, MD, https://seer.cancer.gov/csr/1975_2015/, based on November 2017 SEER data submission, posted to the SEER web site, April 2018.

2. Hospital de SOLCA. Secretaría General. Estadística de los Registro de SOLCA. Guayaquil: Secretaria General del Hospital de SOLCA; 2010- 2015

3. Hogan A, Zhuge Y, Perez E. Pediatric thyroid carcinoma: incidence and outcomes in 1753 patients. J Surg 2009;156:167-172.

4. Spoudeas HA, Harrison B. Paediatric Endocrine Tumours: A Multi-Disciplinary Consensus Statement of Best Practice from a Working Group Convened Under the Auspices of the The British Society of Paediatric Endocrinology \& Diabetes and the United Kingdom Children's Cancer Study Group. The British Society of Paediatric Endocrinology and Diabetes (BSPED) 2005;4:116.

5. Monaco S, Pantanowitz L, Khalbuss W. Cytomorphological and molecular genetic findings in pediatric thyroid fine-needle aspiration. Cancer Cytopathol 2012;120:342-350.

6. Halac I, Zimmerman D. Thyroid nodules and cancers in children. Endocrinol Metab Clin North Am 2005;34:725-744.

7. British Thyroid Association. Royal College of Physicians Guidelines for the Management of Thyroid Cancer. Royal College of Physicians 2007. Second edition, 1:122.

8. Hay I, Gonzalez-Losada T, Reinalda M. Long-term outcome in 215 children and adolescents with papillary thyroid cancer treated during 1940 through 2008. World J Surg 2010;34:1192-1202.

9. NCCN Clinical Practice Guidelines in Oncology (NCCN Guidelines ${ }^{\circledR}$ ) [Internet] . Thyroid Carcinoma. Practice Guidelines in Oncology 2017; Vol 1. Available from: https://www.nccn.org/professionals

10. LaFranchi S. Thyroid cancer in children: Performs a continuous review of over 375 journals and other resources [Internet]. Wolters Kluwer 2007; Available from: www.uptodate.com.

11. American Thyroid Association (ATA) [Internet]. American Thyroid Association. 2017. Available from: https://www.thyroid.org

12. Gharib H, Papini E, Paschke R, Duick DS, Valcavi R, Hegedüs $L$, et al. Medical guidelines for clinical practice for the diagnosis and management of thyroid nodules. American Association of Clinical Endocrinologists and Associazione Medici Endocrinologi. 2006;12(1):63-93.

13. Angelos P. Current Approaches to the Treatment of Well-differentiated Thyroid Cancer. UBM Medica LCC: Oncology 2002;16:3.

14. Demidchik YE, Demidchik EP, Reiners C, Biko J, Mine M, Saenko VA, et al. Comprehensive clinical assessment of 740 cases of surgically treated thyroid cancer in children of Belarus. Anls Surg 2006;4:525532.

15. Handkiewicz-Junak D, Wloch J, Roskosz J, Krajewska J, Kropinska A, Pomorski L, et al. Total Thyroidectomy and Adjuvant Radioiodine Treatment Independently decrease Locoregional recurrence Risk in Childhood and Adolescent Differentiated Thyroid Cancer. J Nucl Med. 2007;48(6): 879-888 